\title{
Management of urticaria and angioedema
}

\section{Paolo Pigatto}

Dipartimento di Tecnologie per la Salute,Clinica Dermatologica,Università degli Studi di Milano \& Ospedale IRCCS R. Galeazzi

This paper is a guidance for management of patients with urticaria and angioedema based on evidence opinion and aimed at adult and paediatrician treatment

The recommendations are evidence graded and included clinical classification, aetiology diagnosis, investigations treatment guidance with special emphasis on children with urticaria and the use of antihistamines in women are pregnant and breastfeeding. 